2. R. H. Bruck, Analogues of the ring of rational integers, Proc. Amer. Math. Soc. vol. 6 (1955) pp. 50-57.

3. I. M. H. Etherington, Non-associative arithmetics, Proceedings of the Royal Society of Edinburgh vol. 62 (1949) pp. 442-453.

4. Trevor Evans, On multiplicative systems defined by generators and relations, I. Normal form theorems, Proc. Cambridge Philos. Soc. vol. 47 (1951) pp. 637-649.

5. - On multiplicative systems defined by generators and relations, II. Monogenic loops, Proc. Cambridge Philos. Soc. vol. 49 (1953) pp. 579-589.

6. Trevor Evans and B. H. Neumann, On varieties of groupoids and loops, J. London Math. Soc. vol. 28 (1953) pp. 342-350.

7. Helene Popova, Sur la logarithmetique d'une boucle, C.R. Acad. Sci. Paris vol. 236 (1953) pp. 1220-1222.

EMORY UNIVERSITY

\title{
COMPONENT SUBSETS OF THE FREE LATTICE ON $n$ GENERATORS
}

\author{
RICHARD A. DEAN
}

1. Introduction. In the free lattice on $n$ generators, $\mathrm{FL}(n)$, the components of a word (lattice polynomial) are defined recursively by: (i) the only component of a generator is itself, and (ii) if $W \equiv A \cup B$ (or $A \cap B$ ) the components of $W$ are $W, A$, and $B$ and their components. ${ }^{1}$ A component subset, $P$, of $\mathrm{FL}(n)$ is a collection of words in $\mathrm{FL}(n)$ with the following property: if a word belongs to $P$ then so do all its components. ${ }^{2}$ A component subset of $\mathrm{FL}(n)$ may be partially ordered in a natural way: $A \geqq B$ if and only if $A \geqq B$ in $\operatorname{FL}(n)$. Clearly if $W \equiv A \cup B$ (or $A \cap B$ ) belongs to a component subset, $P$, $W$ appears in $P$ as the l.u.b. (or g.l.b.) of $A$ and $B$ under the ordering $(\geqq)$. Thus it is natural to say that a component subset $P$ is generated by the generators of $\mathrm{FL}(n)$ which appear in the words belonging to $P$. This notion is used here to prove:

THEOREM $1 .^{8}$ Given any two words, unequal in $\mathrm{FL}(n)$, there exists a finite homomorphic image of $\mathrm{FL}(n)$ in which their images are distinct.

TheOREM 2.4 Any lattice possessing a countable number of generators

Received by the editors May 2, 1955 .

1 (三) denotes logical identity.

2 The author is indebted to R. P. Dilworth for suggesting the definition of component subset and its application in the proof of Theorem 2.

2 Theorem 1 was conjectured by Marshall Hall, Jr.

- Theorem 2 is due to Sorkin [4] where a sketch of a proof is given. 
can be embedded in a lattice with 3 generators.

From the construction given in the proof of Theorem 2, it follows that a finite lattice can be embedded in a lattice with 3 generators.

2. Proof of Theorem 1. Let $A$ and $B$ be two unequal words in $\mathrm{FL}(n)$. Consider the component subset $P$ of $\mathrm{FL}(n)$ formed by $A \cup B$ and its components. Let $x_{1}, \cdots, x_{k}$ be the generators of $\mathrm{FL}(n)$ appearing in $A \cup B$, renumbering if necessary. As indicated in the introduction, $P$ is a partially ordered set generated by $x_{1}, \cdots, x_{k}$ in which $A$ and $B$ appear as distinct elements. Using the MacNeille completion by cuts [3], $P$ may be completed to a lattice $L(P)$. $L(P)$ is an embedding of $P$ which preserves all l.u.b.'s and g.l.b.'s which exist in $P$. In the embedding, let $X \in P$ correspond to $X^{\prime} \in L(P)$. An element $W$ of $P$, not a generator, has the form $W \equiv X \cup Y$ (or $X \cap Y$ ). As remarked in the introduction, $W$ is the l.u.b. (or g.l.b.) of $X$ and $Y$ in $P$ under $(\geqq)$. Hence in $L(P), W^{\prime}=X^{\prime} \cup Y^{\prime}$ (or $X^{\prime} \cap Y^{\prime}$ ). Moreover, the elements added in the completion are just the 1.u.b.'s and g.l.b.'s of elements in $P$, so that $L(P)$ is generated by the $k$ generators, $x_{1}^{\prime}, \cdots, x_{k}^{\prime}$. Since $P$ is embedded in $L(P), A^{\prime} \neq B^{\prime}$ and, by the construction, $L(P)$ is finite since $P$ is finite.

It remains to be shown that there is a homomorphism mapping $\mathrm{FL}(n)$ on to $L(P)$ and $A \rightarrow A^{\prime}$ and $B \rightarrow B^{\prime}$. The desired homomorphism is generated by $x_{i} \rightarrow x_{i}^{\prime}, i=1, \cdots, k-1 ; x_{k+j} \rightarrow x_{k}^{\prime}, j=0, \cdots, n-k$. This mapping can be extended to a homomorphism of $\mathrm{FL}(n)$ onto $\mathrm{L}(P),{ }^{5}$ and since unions and intersections are preserved we have, proceeding inductively, that if $W \in P, W \equiv X \cup Y$, and $X \rightarrow X^{\prime}$ and $Y \rightarrow Y^{\prime}$, then $W \equiv X \cup Y \rightarrow W^{\prime}=X^{\prime} \cup Y^{\prime}$, and dually. In particular, $A \rightarrow A^{\prime}$ and $B \rightarrow B^{\prime}$.

\section{Quotients in FL(3).}

LEMMA 1. In FL(3) with generators $a, b, c$ the six quotients given by $(a \cup b) \cap(a \cup c) / a \cup(b \cap c)$, its cyclical forms and their duals, and the quotient $(a \cup b) \cap(a \cup c) \cap(b \cup c) /(a \cap b) \cup(a \cap c) \cup(b \cap c)$ are mutually exclusive and contain every word whose length, when written in canonical form $^{6}$ is $\geqq 4$.

Proof. These quotients are mutually exclusive because under the homomorphism generated by the distributive law all elements of a particular quotient have a common image and elements in different quotients have distinct images. The second part of the lemma follows

- See, for example, Birkhoff $[1$, p. 30$]$.

- See Whitman [5] for the definition of these terms. 
from Whitman [6], Lemma 1.1 and Theorem 3.

The following table is constructed as a guide for future calculations. Let

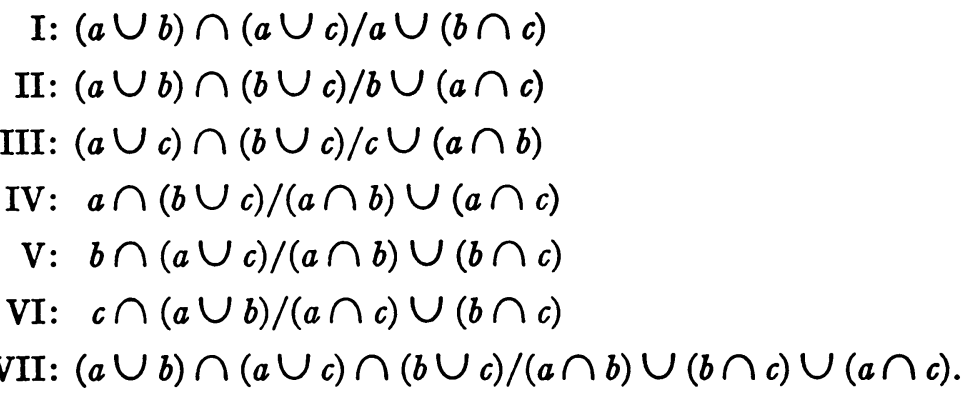

In Table 1 , the entry above the main diagonal in row $i$, column $j$ is $x \cup y$ or the quotient to which $x \cup y$ belongs when $x$ is the element, or belongs to the quotient, heading row $i$ and $y$ is the element, or belongs to the quotient, heading column $j$. Similarly, $x \cap y$ is entered below the main diagonal.

TABLE 1

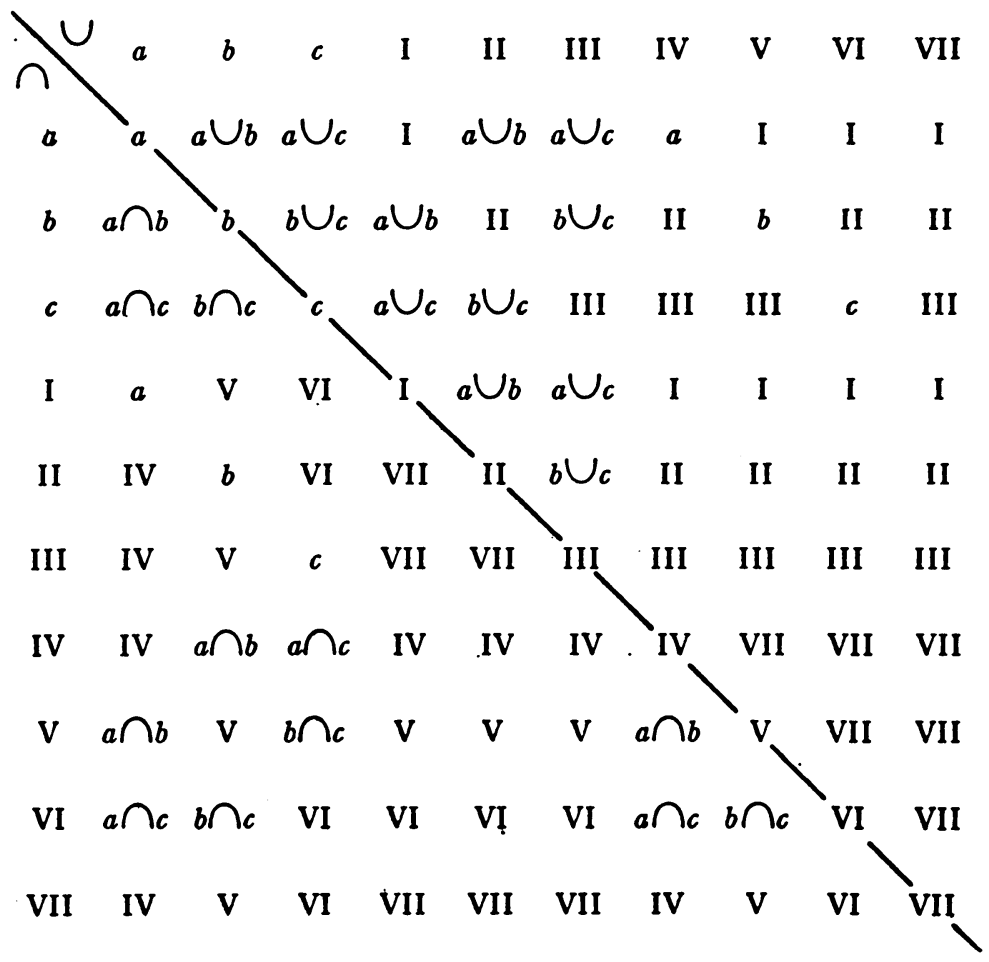


Lemma 2. If $X \in \mathrm{I}$ and $X$ has a canonical form $X \equiv a \cup Y$, then

$$
\mathrm{Y} \in \mathrm{VI} \text { implies } X \cap b \text { is canonical form }
$$

and

$$
\mathrm{Y} \in \mathrm{V} \text { implies } X \cap c \text { is canonical form. }
$$

Proof. (1) shall be proved. The proof of (2) follows by interchanging $b$ and $c$. First observe that $X \cap b \in \mathrm{V}$. Let $X \cap b=W$ where $W$ is in canonical form.

CASE 1. $W \equiv U_{i} W_{i}$. By Corollary 1.2, Whitman [6], $X \cap b=X$ or $b$. Thus $X \geqq b$ or $b \geqq X$, but neither alternative is possible as $X \in \mathrm{I}$.

CASE 2. $W \equiv \bigcap_{i} W_{i}=X \cap b$. By the dual to (19), Whitman [5], $W_{i} \geqq X$ or $b$, for all $i$. If $W_{i} \geqq X$ for all $i$, then $X \cap b \geqq X \geqq X \cap b$. As in Case 1 , this is impossible. Hence $W_{i} \geqq b$ for some $i$. On the other hand $b \geqq \bigcap_{i} W_{i}$, hence $b \geqq W_{j}$ for some $j$. By the assumed canonicity of $W$, it follows that $i=j$, and only one such index exists; let $i=1$. Hence for $i>1, W_{i} \geqq X$. Thus $X \equiv a \cup Y \geqq b \cap \bigcap_{i>1} W_{i} \equiv W$. By (17) Whitman [5], this holds if and only if one of the following hold:

(1) $a \geqq W$, (2) $Y \geqq W$, (3) $X \geqq b$, (4) $X \geqq W_{\text {, }}$, for some $j \geqq 2$. (1) can't hold as $W \in \mathrm{V}$, (2) can't hold as $Y \in \mathrm{VI}, W \in \mathrm{V}$, and (3) can't hold as $X \in \mathrm{I}$. But if (4) holds, and since it is the only alternative left it must hold for some $j \geqq 2, W_{i} \geqq X \geqq W_{j}$, for all $i \geqq 2$. Thus, by the canonicity of $W, i=j$ and only one such index exists. Thus $\bigcap_{i} W_{i}$ $\equiv X \cap b$ is the canonical form of $W$. Cyclical and dual lemmas hold.

4. Proof of Theorem 2. Let $L$ have $n$ generators $g_{i}, i=1,2,3, \cdots$. In $\mathrm{FL}(3)$ consider the generators of a sublattice isomorphic to $\mathrm{FL}(2 n)$. In particular, select those generators, $a_{m}$, given by Dilworth [2]. Let $F$ denote the component subset of FL(3) formed by the $a_{m}, m=1$, $2, \cdots$, and their components. Thus the elements of $F$ and the quotients to which they belong are $X_{0}=a$, and for $m=1,2, \cdots$ :

$$
\begin{array}{rlrl}
\alpha_{m} \equiv c \cap X_{m-1} \in \mathrm{VI}\left(\alpha_{1}=a \cap c\right), \\
\alpha_{-m} \equiv c \cup X_{-m+1} \in \mathrm{III}\left(\alpha_{-1}=a \cup c\right), \\
\beta_{m} \equiv b \cup \alpha_{m} \in \mathrm{II}, & \beta_{-m} \equiv b \cap \alpha_{-m} \in \mathrm{V}, \\
\gamma_{m} \equiv a \cap \beta_{m} \in \mathrm{IV}, & \gamma_{-m} \equiv a \cup \beta_{-m} \in \mathrm{I}, \\
\delta_{m} \equiv c \cup \gamma_{m} \in \mathrm{III}, & \delta_{-m} \equiv c \cap \gamma_{-m} \in \mathrm{VI}, \\
\epsilon_{m} \equiv b \cap \delta_{m} \in \mathrm{V}, & \epsilon_{-m} \equiv b \cup \delta_{-m} \in \mathrm{II}, \\
X_{m} \equiv a \cup \epsilon_{m} \in \mathrm{I}, & X_{-m} \equiv a \cap \epsilon_{-m} \in \mathrm{IV}, \\
\mu_{m} \equiv X_{m} \cap \lambda_{-m} \in \mathrm{VII}, & \lambda_{-m} \equiv c \cup X_{-m} \in \mathrm{III}\left(=\alpha_{-m-1}\right), \\
a_{m} \equiv b \cup \mu_{m} \in \mathrm{II} . &
\end{array}
$$


LEMMA 3. The elements of $F$ are in canonical form.

Proof. By applying Lemma 2 and its cyclical and dual forms it is clear that $\alpha_{m}, \beta_{m}, \gamma_{m}, \delta_{m}, \epsilon_{m}, X_{m}$ and $\lambda_{m}, m= \pm 1, \pm 2, \cdots$, are in canonical form. The canonicity of $\mu_{m}$ and $a_{m}$ is established by verifying Whitman's criteria, [6, Corollary 1.1].

For $\mu_{m}$, it is clear that $X_{m}$ and $\lambda_{-m}$ are in canonical form and noncomparable. $X_{m} \cap \lambda_{-m} \equiv\left(a \cup \epsilon_{m}\right) \cap\left(X_{-m} \cup c\right)$ and since $a \geq \mu_{m}, \epsilon_{m} \geq \mu_{m}$, $X_{-m} \geq \mu_{m}$, and $c \geq \mu_{m}$, it follows that $\mu_{m}$ must be in canonical form.

For $a_{m} \equiv b \cup_{\mu_{m}} \equiv b \cup\left(X_{m} \cap \lambda_{-m}\right)$, it is clear that $b$ and $\mu_{m}$ are in canonical form and noncomparable and, since $X_{m} \geq a_{m}$ and $\lambda_{-m} \geq a_{m}$, hence $a_{m}$ is in canonical form.

Proceeding with the proof of Theorem 2, the elements of $L$ and $F$ are combined into a single set $P=[L, F]$ which is ordered as follows:

Definition 1. For $X$ and $Y \in P, X \supseteq Y$ if and only if one or more of the following hold:

(1) $X, Y \in L$ and $X \geqq Y$ in $L$.

(2) $X, Y \in F$ and $X \geqq Y$ in $\mathrm{FL}(3)$.

(3) $X=g_{m}$ and $Y=a_{2 m-1}$ or $a_{2 m}, m=1,2, \cdots$.

(4) $X \in L, Y \in F$ and $X \geqq U_{1} \cap \cdots \cap U_{r}$ in $L$ and for every $i$ there is a $j$ such that $U_{i} \geqq g_{j}$ and $a_{2 j-1}$ or $a_{2 j} \geqq Y$ in $\mathrm{FL}(3)$.

The proof of Theorem 2 rests on the following lemmas.

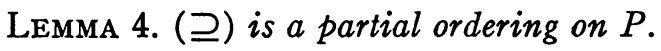

LEMMA 5. () preserves unions and intersections existing in $L$.

LemMa 6. No element of $F$ contains $a_{m}$ except $a_{m}$.

LEMMA 7. $g_{i}$ is the l.u.b. under $\supseteq$ of $a_{2 i-1}$ and $a_{2 i}$.

LEMMA 8. () preserves unions and intersections in $F$ which hold in FL(3).

The proof of the Theorem 2 now follows directly. Using the MacNeille completion by cuts [3], complete the partially ordered set $P=[L, F, \supseteq]$ to a lattice $L(P)$. Since by Lemma 5 , ( $)$ preserves unions and intersections in $L, L(P)$ is an embedding for $L$. Since $a, b, c$ generate $F$ and, by Lemma $7, g_{m}$ is the l.u.b. of $a_{2 m-1}$ and $a_{2 m}$ in $P$, and since, by Lemma $8,(\supseteq)$ preserves unions and intersections in $F, a, b, c$, generate $P$ and hence $L(P)$ also. Finally, $P$ is finite if $L$ is finite. Thus the following corollary is proved.

Corollary. Any finite lattice can be embedded in a finite lattice with three generators.

The proofs of Lemmas 4 and 5 are left to the reader. Lemma 7 fol- 
lows immediately from Lemma 6 and Definition 1. Lemmas 6 and 8 will be proved in detail.

Proof of Lemma 6. Since $a_{m} \in I I$, the only candidates for $X$ such that $X \geqq a_{m}$ are $\epsilon_{-r}, \beta_{r}, a_{r} . a_{r} \geqq a_{m}$ if and only if $r=m$, since the $a_{m}$ are noncomparable. Suppose that $\epsilon_{-r} \geqq a_{m}$. This holds if and only if $\epsilon_{-r} \geqq \mu_{m}$. This last relation holds in FL(3) if and only if one of the following hold:
(1) $\epsilon_{\rightarrow r} \geqq X_{m}$,
(2) $\epsilon_{-r} \geqq \lambda_{-m}$,
(3) $b \geqq \mu_{m}$,
(4) $\delta_{\rightarrow} \geqq \mu_{m}$.

However none of these can hold since the elements belong to the wrong quotients - see Table 1.

Suppose that $\beta_{r} \geqq a_{m}$. This holds if and only if $\beta_{r} \geqq \mu_{m}$. Again one of four possibilities must hold:
(1) $\beta_{r} \geqq X_{m}$,
(2) $\beta_{r} \geqq \lambda_{-m}$,
(3) $b \geqq \mu_{m}$,
(4) $\alpha_{r} \geqq \mu_{m}$.

As before, none of these can be true.

Proof of Lemma 8. Part 1. Suppose $W, X, Y \in F$ and $W=X \cap Y$ in FL(3). Then $X$ and $Y \geqq W$, hence $X$ and $Y \supseteq W$ in $P$. Thus $W$ is a lower bound for $X$ and $Y$. Let $X$ and $Y \supseteq Z$ in $P$. Since $X$ and $Y \in F, Z \in F$ also, as no element of $F$ can contain ( $\supseteq$ ) an element of $L$, by definition. Thus $X$ and $Y \geqq Z$, whence $W=X \cap Y \geqq Z$ and thus $W \supseteq Z$.

PART 2. Suppose $W, X, Y \in F$ and $W=X \cup Y$ in $F L(3)$. By the dual of the first argument in Part $1, W$ is an upper bound for $X$ and $Y$ under $\supseteq$. Suppose $Z \supseteq X$ and $Y$. If $Z \in F$ the dual of the second argument in Part 1 shows that $Z \supseteq W$. Suppose that $Z \in L$. Now $Z \supseteq X, Y$ and $X, Y \in F$ imply that there exist indices $r$ and $s$ such that $a_{r} \geqq X$ and $a_{s} \geqq Y$. Thus $X$ and $Y$ must come from quotients II, IV, V, VI, VII, or $=b$. From a study of Table $1, X \cup Y$ must then belong to quotients II, VII, IV (if and only if $X$ and $Y \in \mathrm{IV}$ ), $\mathrm{V}$ (if and only if $X$ and $Y \in \mathrm{V}$ ), VI (if and only if $X$ and $Y \in \mathrm{VI}$ ), or $=b$.

If $X$ and $Y$ both belong to $\mathrm{V}$ or $X \cup Y=b$, then every $a_{m} \geqq X \cup Y$ and in this case $Z \supseteq X \cup Y$.

When $W=X \cup Y \in \mathrm{IV}, \mathrm{VI}$, or VII, it will be shown that $W=X \cup Y$ $=X$ or $Y$ and thus $Z \supseteq X$ and $Y$ implies trivially that $Z \supseteq W=X \cup Y$. Since $W=X \cup Y \in I V$, VI, or VII, $W$ must have the canonical form $p \cap q$ where $p$ and $q$ are generators or intersections. Now $W=X \cup Y$ $\equiv \cup_{i} T_{i}$ where each $T_{i}$ appears in $F$ as a generator or as an intersection. From the dual of Corollary 1.2, Whitman [6], $W=X \cup Y$ $\equiv \mathrm{U}_{i} T_{i}=T_{k}$ for some index $k$. Thus since $X$ and $Y$ are unions of certain of the $T_{i}, X$ or $Y \geqq T_{k}$. But $T_{k} \geqq X$ and $Y$. Thus $W=T_{k}=X$ or $Y$.

When $W=X \cup Y \in I I, W$ must have the canonical form $b \cup p$ where $p$ is an intersection. Thus $W=X \cup Y \equiv \bigcup_{i} T_{i}$ where each $T_{i}$ appears 
in $F$ as a generator or as an intersection. From (19) Whitman [5], $b \leqq T_{h}$ and $p \leqq T_{k}$ for some $h$ and $k$. Thus $W \equiv b \cup_{p} \leqq T_{h} \cup T_{k} \leqq \cup_{i} T_{i}$ $=X \cup Y=W$. Since $X$ and $Y$ are unions of certain of the $T_{i}, X$ or $Y \geqq T_{h}$ and $X$ or $Y \geqq T_{k}$. If either $X$ or $Y$ contains both $T_{h}$ and $T_{k}$, then $X$ and $Y$ are comparable in $F$ so that $Z \supseteq X$ and $Y$ implies trivially that $Z \supseteq W=X \cup Y$.

Suppose then that $X \geqq T_{h} \geqq b$ and $Y \geqq T_{k} \geqq p$. Thus $Z \supseteq Y$ implies $Z \supseteq p$. Now $Z \supseteq Y$ and $Z \in L$ implies that $Z \geqq u_{1} \cap \cdots \cap u_{r}$ in $L$ where for each index $i$ there exists a $j$ such that $u_{i} \geqq g_{j}$ and either $a_{2 j-1}$ or $a_{2 j} \geqq Y \geqq p$ in FL(3). But every $a_{m} \geqq b$. Thus either $a_{2 j-1}$ or $a_{2 j} \geqq b \cup p$. Thus $Z \supseteq W=X \cup Y=b \cup p$.

\section{BIBLIOGRAPHY}

1. G. Birkhoff, Lattice theory, rev. ed., Amer. Math. Soc. Colloquium Publications, vol. 25, New York, 1948.

2. R. P. Dilworth, Lattices with unique complements, Trans. Amer. Math. Soc. vol. 57 (1945) pp. 123-154.

3. H. M. MacNeille, Partially ordered sets, Trans. Amer. Math. Soc. vol. 42 (1937) pp. 416-460.

4. Yu I. Sorkin, On the imbedding of latticoids in lattices, Doklady Akademii Nauk SSSR (N.S.) vol. 95 (1954) pp. 931-934 (Russian).

5. P. Whitman, Free lattices I, Ann. of Math. (2) vol. 42 (1941) pp. 325-330.

6. - Free lattices II, Ann. of Math. (2) vol. 43 (1942) pp. 104-115.

Califoraia Institute of Technology 